\title{
The Motion of a Bead Sliding on a Wire in Fractional Sense
}

\author{
D. BALEANU ${ }^{a, b}$, A. JAJARMi ${ }^{c}$, J.H. AsAD ${ }^{d, *}$ AND T. BlaszCZYK ${ }^{e}$ \\ ${ }^{a}$ Department of Mathematics and Computer Science, Faculty of Arts and Sciences, Cankaya University, \\ 06530 Ankara, Turkey \\ ${ }^{b}$ Institute of Space Sciences, P.O. Box MG-23, 76900, Magurele, Bucharest, Romania \\ ${ }^{c}$ Department of Electrical Engineering, University of Bojnord, P.O. Box 94531-1339, Bojnord, Iran \\ ${ }^{d}$ Department of Physics, College of Arts and Sciences, Palestine Technical University, P.O. Box 7, Tulkarm, Palestine \\ ${ }^{e}$ Institute of Mathematics, Czestochowa University of Technology,
} al. Armii Krajowej 21, 42-201 Częstochowa, Poland

(Received February 26, 2017)

In this study, we consider the motion of a bead sliding on a wire which is bent into a parabola form. We first introduce the classical Lagrangian from the system model under consideration and obtain the classical EulerLagrange equation of motion. As the second step, we generalize the classical Lagrangian to the fractional form and derive the fractional Euler-Lagrange equation in terms of the Caputo fractional derivatives. Finally, we provide numerical solution of the latter equation for some fractional orders and initial conditions. The method we used is based on a discretization scheme using a Grünwald-Letnikov approximation for the fractional derivatives. Numerical simulations verify that the proposed approach is efficient and easy to implement.

DOI: 10.12693/APhysPolA.131.1561

PACS/topics: motion of a bead on a wire, Euler-Lagrange equation, fractional derivative, Grünwald-Letnikov approximation

\section{Introduction}

Fractional calculus plays an important rule in describing complex behaviors of many real life phenomena $[1,2]$. It has been demonstrated that the fractional order representation provides a more realistic behavior of complex systems appearing in various fields of science and engineering [3]. Due to this fact, fractional calculus has some applications in chemistry [4], bioengineering [5], hydrologic modelling [6], pharmacokinetics [7], heat transfer modelling [8], viscoelasticity [9], etc. In classical mechanics fractional calculus finds a wide range of applications, specially problems involving Lagrangian and Hamiltonian. The first attempt to study non-conservative Lagrangian and Hamiltonian mechanics within fractional calculus was carried by Riewe $[10,11]$. Following Riewe's work, many efforts have been carried out [12-15]. In these works, the system under consideration is described by the fractional Lagrangian or Hamiltonian equations, and as a result the fractional Euler-Lagrange equations (FELEs) or the fractional Hamilton equations are derived for the considered problems. The obtained fractional equations cannot be solved analytically so easily in many cases; therefore we seek for the numerical schemes used for solving fractional differential equations (FDEs). These methods include the Grünwald-Letnikov approximation [2], decomposition method [16-19], variational iteration method [20], the Adams-Bashforth-Moulton method [21], etc.

Within the environment, the analysis of the motion of particles on different surfaces has become a strong topic

${ }^{*}$ corresponding author; e-mail: drjasad@yahoo.com to be considered. Some examples of these phenomena can be found in centrifugation, centrifugal filters and industrial hopper. The surface of motion can take many shapes like circular, conical or parabolic. The particle may also be considered as a bead, dust, bug and so on. In this study, we consider a bead sliding on a wire, that is bent into a parabola shape. The analytical and numerical solutions for this motion can be of great usefulness to analyze the motion of particles on these surfaces (see for example [22] and the references therein). In addition, we believe that the numerical solution of the FELE for the motion of a bead on the wire will reveal new aspects of the non-locality of this system. From the mathematical and practical points of view, the FELEs are new, and their numerical and exact solutions contain more information than the corresponding integer ones. Therefore, by modeling the classical Lagrangian of a bead's motion on a wire with fractional derivatives, we have a class of new solutions. This means that we can easily construct a real model describing the motion of a bead on the wire corresponding to the new FELE. This point can be considered as one of the major advantages of the fractional calculus versus the classical one, because using the nonlocal fractional differential operators enables us to build a new real world phenomenon, and we are not violating any existing laws based on the classical calculus approach.

The outline of this paper is as follows. In Sect. 2 some preliminaries concerning fractional derivatives are presented. In Sect. 3, classical and fractional studies have been carried out for a bead sliding a wire. Section 4 provides numerical solutions of the derived FELE for different values of fractional order and initial conditions. Finally, we close the paper by a conclusion in Sect. 5 . 


\section{Preliminaries}

This section gives briefly some preliminaries concerning the fractional derivatives. There are several definitions for a fractional derivative including RiemannLiouville, Weyl, Caputo, Marchaud, and Riesz [1, 2]. Here, we define the left and right fractional derivatives in terms of the Riemann-Liouville and Caputo. Let $g:[a, b] \rightarrow R$ be a time dependent function. Below, we define the fractional derivatives of $g(t)$ used in this manuscript.

The left Riemann-Liouville fractional derivative (LRLFD):

$$
\begin{aligned}
&{ }_{a} D_{t}^{\alpha} g(t)=\frac{1}{\Gamma(n-\alpha)} \\
& \times\left(\frac{\mathrm{d}}{\mathrm{d} t}\right)^{n} \int_{a}^{t}(t-\tau)^{n-\alpha-1} g(\tau) \mathrm{d} \tau .
\end{aligned}
$$

The right Riemann-Liouville fractional derivative (RRLFD):

$$
\begin{aligned}
& { }_{t}^{C} D_{b}^{\alpha} g(t)=\frac{1}{\Gamma(n-\alpha)} \\
& \quad \times\left(-\frac{\mathrm{d}}{\mathrm{d} t}\right)^{n} \int_{t}^{b}(t-\tau)^{n-\alpha-1} g(\tau) \mathrm{d} \tau .
\end{aligned}
$$

The left Caputo fractional derivative (LCFD):

$$
\begin{aligned}
& { }_{a}^{C} D_{t}^{\alpha} g(t)=\frac{1}{\Gamma(n-\alpha)} \\
& \quad \times \int_{a}^{t}(t-\tau)^{n-\alpha-1}\left(\frac{\mathrm{d}}{\mathrm{d} \tau}\right)^{n} g(\tau) \mathrm{d} \tau,
\end{aligned}
$$

and the right Caputo fractional derivative (RCFD):

$$
\begin{aligned}
& { }_{t}^{C} D_{b}^{\alpha} g(t)=\frac{1}{\Gamma(n-\alpha)} \\
& \quad \times \int_{t}^{b}(t-\tau)^{n-\alpha-1}\left(-\frac{\mathrm{d}}{\mathrm{d} \tau}\right)^{n} g(\tau) \mathrm{d} \tau,
\end{aligned}
$$

where $\Gamma(\cdot)$ is the Euler Gamma function and $n-1<\alpha<n$ is the fractional derivative order. In addition, when $\alpha$ is an integer, the fractional derivatives of Riemann-Liouville and Caputo both coincide with the ordinary derivative $[1,2]$

$$
\begin{aligned}
& { }_{a} D_{t}^{n} g(t)={ }_{a}^{C} D_{t}^{n} g(t)=g^{(n)}(t), \\
& { }_{t}^{C} D_{b}^{\alpha} g(t)={ }_{t}^{C} D_{b}^{\alpha} g(t)=(-1)^{n} g^{(n)}(t) .
\end{aligned}
$$

For more details on the fractional derivatives and their properties, the readers can refer to $[1-3]$.

\section{Classical and fractional studies for the motion of a bead on a wire}

We start by describing our physical system. Consider a wire that is bent into a parabola shape $y=A x^{2}$ with axis vertical in the Earth gravitational field $g$, a bead of mass $m$ is sliding without friction along the wire. The kinetic and potential energy of the bead respectively are

$$
\begin{aligned}
T & =\frac{1}{2} m\left(\dot{x}^{2}+\dot{y}^{2}\right)=\frac{1}{2} m\left[1+4 A^{2} x^{2}\right] \dot{x}^{2}, \\
V & =m g y=m g A x^{2} .
\end{aligned}
$$

As a result the traditional Lagrangian takes the form

$$
L(x, \dot{x}, t)=T-V=\frac{1}{2} m\left(1+4 A^{2} x^{2}\right) \dot{x}^{2}-m g A x^{2},(9)
$$

and the classical Euler-Lagrange equation (CELE) is obtained from

$$
\frac{\partial L}{\partial x}-\frac{\mathrm{d}}{\mathrm{d} t} \frac{\partial L}{\partial \dot{x}}=0
$$

In view of Eqs. (9),(10) we obtain the CELE

$$
\ddot{x}\left(m+4 m A^{2} x^{2}\right)+\dot{x}^{2}\left(4 m A^{2} x\right)+2 m g A x=0 .
$$

In the following, we investigate the fractional form of the classical Lagrangian (Eq. (9)) which reveals new aspects of the physical system under consideration

$$
L^{F}=\frac{1}{2} m\left(1+4 A^{2} x^{2}\right)\left({ }_{a}^{C} D_{t}^{\alpha} x\right)^{2}-m g A x^{2} .
$$

Then the FELE of motion is obtained from

$$
\frac{\partial L^{F}}{\partial x}+{ }_{t}^{C} D_{b}^{\alpha} \frac{\partial L^{F}}{\partial_{a}^{C} D_{t}^{\alpha} x}+{ }_{a}^{C} D_{t}^{\beta} \frac{\partial L^{F}}{\partial_{t}^{C} D_{b}^{\alpha} x}=0 .
$$

As a result of using Eqs. (12),(13) the FELE reads

$$
\begin{gathered}
4 m A^{2} x\left({ }_{a}^{C} D_{t}^{\alpha} x\right)^{2}-2 m g A x+m_{t}^{C} D_{b a}^{\alpha C} D_{t}^{\alpha} x \\
+4 m A_{t}^{2 C} D_{b}^{\alpha}\left(x_{a}^{2 C} D_{t}^{\alpha} x\right)=0 .
\end{gathered}
$$

Notice that as $\alpha \rightarrow 1$, the FELE (14) reduces to the CELE (11).

Below, we are going to obtain the fractional Hamilton equation of motion. For this purpose, let us introduce the following generalized momenta

$$
\begin{aligned}
& P_{\alpha}=\frac{\partial L^{F}}{\partial_{a}^{C} D_{t}^{\alpha} x}=m\left(1+4 A^{2} x^{2}\right)_{a}^{C} D_{t}^{\alpha} x, \\
& P_{\beta}=\frac{\partial L^{F}}{\partial_{t}^{C} D_{b}^{\alpha} x}=0 .
\end{aligned}
$$

Then the fractional Hamiltonian function can be obtained from

$$
H^{F}\left(x, P_{\alpha}, P_{\beta}, t\right)=P_{\alpha a}^{C} D_{t}^{\alpha} x+P_{\beta t}^{C} D_{b}^{\beta} x-L^{F},
$$

which implies that

$$
H^{F}\left(x, P_{\alpha}, P_{\beta}, t\right)=\frac{1}{2 m\left(1+4 A^{2} x^{2}\right)} P_{\alpha}^{2}+m g A x^{2} .
$$

The fractional Hamilton equation of motion reads

$$
\frac{\partial H^{F}}{\partial x}={ }_{t}^{C} D_{b}^{\alpha} P_{\alpha}+{ }_{a}^{C} D_{t}^{\alpha} P_{\beta},
$$

which results again the FELE (14). In the next section, we aim to solve Eq. (14) numerically for some fractional orders and initial conditions.

\section{Numerical solution and simulation results}

To develop an approximation scheme for the FELE (14), first we reformulate Eq. (14) in the following way. Let us define the new state variable $\eta(t)$ as $\eta(t):=$ ${ }_{a}^{C} D_{t}^{\alpha} x(t)$. Then Eq. (14) can be rewritten in the form of the following system of FDEs:

$$
\left\{\begin{array}{l}
{ }_{a}^{C} D_{t}^{\alpha} x(t)=\eta(t), \\
{ }_{t}^{C} D_{b}^{\alpha} \eta(t)+4 A_{t}^{2 C} D_{b}^{\alpha} p(t)=2 g A x(t)-4 A^{2} x(t) \eta^{2}(t)
\end{array}\right.
$$

where $p(t)=x^{2}(t) \eta(t)$. Now, we consider a uniform mesh on $[a, b]$ and label the nodes $0,1, \ldots, N$ where $N$ is an arbitrary positive integer and $h_{N}=\frac{b-a}{N}$ is the time step 
size. We denote $\left(x_{i}, \eta_{i}, p_{i}\right)$ as the numerical approximation of $\left(x\left(t_{i}\right), \eta\left(t_{i}\right), p\left(t_{i}\right)\right)$ where $t_{i}=a+i h_{N}$ is the time at node $i$ for $0 \leq i \leq N$. Using the Grünwald-Letnikov approximations for the Caputo fractional derivatives in Eq. (14), we have the following formulae:

$$
\begin{aligned}
\left.{ }_{a}^{C} D_{t}^{\alpha} x(t)\right|_{t=t_{i}} & =h_{N}^{-\alpha} \sum_{j=0}^{i} \omega_{j}^{(\alpha)} x_{i-j} \\
-\frac{1}{\Gamma(1-\alpha)} \frac{x_{0}}{\left(t_{i}-t_{0}\right)^{\alpha}}, & \\
\left.{ }_{t}^{C} D_{b}^{\alpha} \eta(t)\right|_{t=t_{i}} & =h_{N}^{-\alpha} \sum_{j=0}^{N-i} \omega_{j}^{(\alpha)} \eta_{i+j} \\
-\frac{1}{\Gamma(1-\alpha)} & \frac{\eta_{N}}{\left(t_{N}-t_{i}\right)^{\alpha}}, \\
\left.{ }_{t}^{C} D_{b}^{\alpha} p(t)\right|_{t=t_{i}} & =h_{N}^{-\alpha} \sum_{j=0}^{N-i} \omega_{j}^{(\alpha)} p_{i+j} \\
-\frac{1}{\Gamma(1-\alpha)} & \frac{p_{N}}{\left(t_{N}-t_{i}\right)^{\alpha}},
\end{aligned}
$$

where the binomial coefficient $\omega_{j}^{(\alpha)}$ can be calculated by using the recursive formula $\omega_{j}^{(\alpha)}=\left(1-\frac{\alpha+1}{j}\right) \omega_{j-1}^{(\alpha)}$ for $\omega_{0}^{(\alpha)}=1$. Substituting Eqs. (21)-(23) into Eq. (20), the following system of nonlinear algebraic equations is obtained for the FELE (14):

$$
\left\{\begin{array}{l}
h_{N}^{-\alpha} \sum_{j=0}^{i} \omega_{j}^{(\alpha)} x_{i-j}-\frac{1}{\Gamma(1-\alpha)} \frac{x_{0}}{\left(t_{i}-t_{0}\right)^{\alpha}}=\eta_{i}, \\
h_{N}^{-\alpha} \sum_{j=0}^{N-i} \omega_{j}^{(\alpha)}\left(\eta_{i+j}+4 A^{2} p_{i+j}\right) \\
\quad-\frac{1}{\Gamma(1-\alpha)} \frac{\left(\eta_{N}+4 A^{2} p_{N}\right)}{\left(t_{N}-t_{i}\right)^{\alpha}}=2 g A x_{i}-4 A^{2} x_{i} \eta_{i}^{2},
\end{array}\right.
$$

where $p_{i}=x_{i}^{2} \eta_{i}$.

\section{Simulation results}

In the following simulations we choose $m=2$ and $g=9.81$. We also take the initial condition for $\eta(t)$ as $\eta(0)=0$. In Figs. 1-3 the graphs of $x(t)$ and $y(t)=$ $A x^{2}(t)$ are plotted for $\alpha=0.8,0.85,0.9,0.95,1$ and different values of $x(0)$ and $A$. In these figures, we also pro-
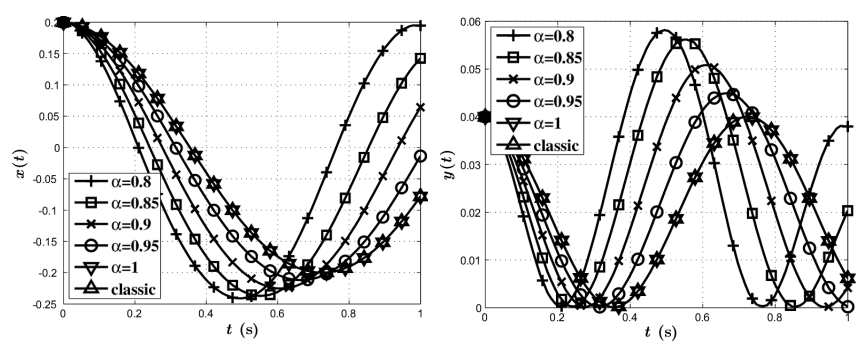

Fig. 1. Simulation curves of $x(t)$ and $y(t)=A x^{2}(t)$ for $A=1, x(0)=0.2$ and different values of $\alpha$.

vide the solution of CELE (11) in addition to some different solutions of FELE (14) for $0<\alpha \leq 1$. These figures indicate that the numerical solution of Eq. (14) exhibits complex behaviors for different values of $\alpha, x(0)$ and $A$. Thus, taking into account the FELEs provides more flexible models which help us to adjust better the dynamical behaviors of many real world systems. Figures 1-3 also verify that the numerical solution of FELE (14) approaches the classical case as $\alpha$ approaches 1 .
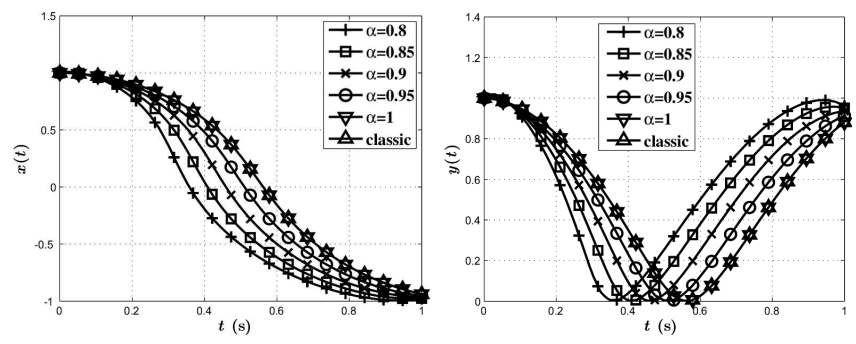

Fig. 2. Simulation curves of $x(t)$ and $y(t)=A x^{2}(t)$ for $A=1, x(0)=1$ and different values of $\alpha$.
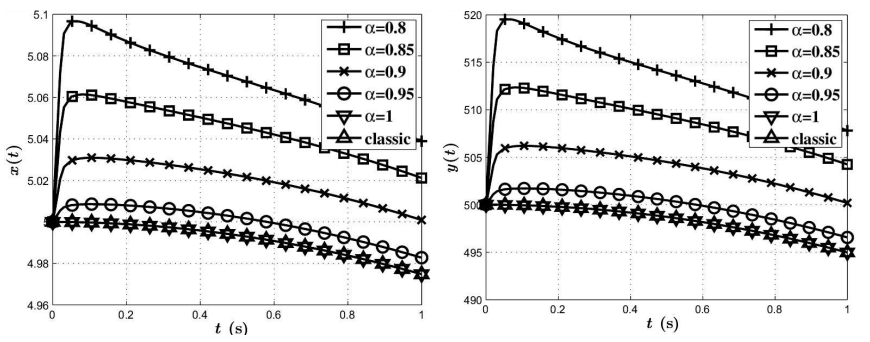

Fig. 3. Simulation curves of $x(t)$ and $y(t)=A x^{2}(t)$ for $A=20, x(0)=5$ and different values of $\alpha$.

\section{Conclusion}

Fractional calculus is an efficient tool to describe complex behaviors of many real world systems. The numerical analysis of FDEs is a very important issue to be considered by researchers. In this work, we discussed the motion of a bead on a wire by the fractional calculus, where we used the fractional derivative in the Caputo sense. We solved the FELE numerically by using a discretization technique based on a Grünwald-Letnikov approximation for the fractional derivative. Simulation results for different values of $\alpha, x(0)$ and $A$ were shown in Figs. 1-3. In these figures, the classic solution of Eq. (11) was also plotted in addition to some different solutions of FELE (14) for $0<\alpha \leq 1$. From these figures it is observed that the behaviors of the FELE depend on the fractional derivative order $\alpha$. Thus, taking into account the fractional calculus provides more flexible models demonstrating new aspects of real world phenomena. Moreover, the numerical results in fractional sense approach the classic solution as $\alpha$ approaches 1 . Numerical simulations verified that the proposed approach is efficient for the fractional equation of motion.

\section{References}

[1] D. Baleanu, J.A.T. Machado, A.C.J. Luo, Fractional Dynamics, Control, Springer Science \& Business Media, 2011.

[2] A.A. Kilbas, H.M. Srivastava, J.J. Trujillo, Theory and Applications of Fractional Differential Equations, Elsevier, Amsterdam 2006. 
[3] D. Baleanu, T. Blaszczyk, J. Asad, M. Alipour, Acta Phys. Pol. A 130, 688 (2016).

[4] J.W. Kirchner, X. Feng, C. Neal, Nature 403, 524 (2000).

[5] R.L. Magin, Crit. Rev. Biomed. Eng. 32, 1 (2004).

[6] D.A. Benson, M.M. Meerschaert, J. Revielle, Adv. Water Res. 51, 479 (2013).

[7] J.K. Popovic, D.T. Spasic, J. Tosic, J. Kolarovic, J.L. Malti, R. Mitic, I.M. Pilipovic, S. Atanack, Commun. Nonlin. Sci. Numer. Simulat. 22, 451 (2015).

[8] D. Sierociuk, A. Dzielinski, G. Sarwas, I. Petras, I. Podlubny, T. Skovranek, Philos. Trans. R. Soc. A 371, 20120146 (2013).

[9] S. Larsson, M. Racheva, F. Saedpanah, Comput. Meth. Appl. Mech. Eng. 283, 196 (2015).

[10] F. Riewe, Phys. Rev. E 53, 1890 (1996).

[11] F. Riewe, Phys. Rev. E 55, 3581 (1997).

[12] O.P. Agrawal, J. Math. Anal. Appl. 272, 368 (2002).
[13] O.P. Agrawal, J. Gregory, K.P. Spector, J. Math. Ann. Appl. 210, 702 (1997).

[14] O.P. Agrawal, J. Vib. Acoust. 126, 561 (2004).

[15] D. Baleanu, S. Muslih, Phys. Scr. 27, 105 (2005).

[16] D. Baleanu, I. Petras, J.H. Asad, M. Pilar, Int. J. Theor. Phys. 51, 1253 (2012).

[17] D. Baleanu, J.H. Asad, I. Petras, Rom. Rep. Phys. 64, 907 (2012).

[18] D. Baleanu, J.H. Asad, I. Petras, S. Elagan, A. Bilgen, Rom. Rep. Phys. 64, 1171 (2012).

[19] D. Baleanu, J.H. Asad, I. Petras, Commun. Theor. Phys. 61, 221 (2014).

[20] S. Momani, Z. Odibat, A. Alawneh, Numer. Meth. Part. D. E. J. 24, 262 (2008).

[21] K. Diethelm, N.J. Ford, A.D. Freed, Nonlin. Dyn. 29, 3 (2002).

[22] M. Hatami, D.D. Ganji, Powder Technol. 258, 94 (2014). 